a wineglassful of water with each dose. 'The addition of camphor mouobromate seems useful, and sometimes a small quantity of quinin, say $1 / 8$ to $1 / 2$ of a grain, may also be added to each dose, although this has not the same applicability in hay fever as in ordinary coryzas.

In many cases it will also be found useful to administer the so-called antilithic remedies, as piperazin quinate, lithium citrate and sodium salicylate. In a few instances i have found a combination of sodium salicylate and sodjum benzoate beneficial.

The following formulas are illustrative:

10. Piperazin quinate

Lithium citrate

Sodium salicylate (true) of each. gr. ii 20

Mix. For one capsule. Sign: One capsule with half a tumblerful of water, after meals and at bedtime (4 times a day).

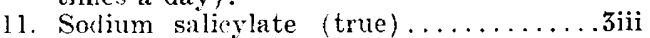

Sodium benzoate (true) ............

Essence of pepsin............f fii

Tincture of nux vomica..........f3i

12

24
60

Peppermint water, sufficient to make...f 3 iv 120

Sign: 2 fluidrams (teaspoonfuls) in (or with) onehalf tumblerful of water, every fourth hour, for four (or five) doses daily.

Mild alkaline carbonated mineral waters are always helpful and patients should be advised to take freely during the hay fever season any palatable water of these groups; for example, one of the numerous American lithia or "Vichy" waters or imported Vichy (Celestin), or Giesshuebl or Fachingen. If preferred, tablets of lithium carbonate or lithium citrate may be used to make an artificial mineral water.

\section{ASTHMATIC PAROXYSMS.}

If the palliative measures already described and the general precautions to be set forth in conclusion are carried out systematically, asthma is not likely to occur. To relieve an attack, a tablet of suprarenalin may be placed on the tongue (1/10 grain, repeated, if necessary, every 10 to 20 minutes) ; or suprarenalin or adrenalin solution $(1: 1000)$ may be applied to the nose by spray or cotton wad; but a hypodermatic injection of 10 to 20 minims of adrenalin or suprarenalin solution (1:1000) is usually the quickest method. If for any reason the suprarenalin preparations are contraindicated, chloroform or ethyl chlorid may be inhaled with due care and not to the induction of general anesthesia. Patients very prone to asthma may be given prophylactic inhalations of ethyl iodid ( 3 to 10 minims every 3 to 4 hours); or one of the following prescriptions may be taken:

12. Aspidospermin hydrochlorate .......gr. iii Hyoscin hydrobromate ............ $1 / 100$

Peruvian balsam ...............sufficient Mix. Make 24 capsules.

Sign: One every hour for 10 to 12 hours daily, or as needed.

13. Morphin hydrochlorate .........gr. 1/6 Strychnin sulphate ............gr. 1/30 Hyoscin hydrobromate .......gr. 1/250 Atropin sulphate ............... 1/250 Sterilized water ............... $x$ Dissolve. Sign: Use by hypodermic injection.

14. Codein sulphate ...............gr. ii 112 Hyoscin hydrobromate ..........gr. $1 / 40$. 1002 Mix. Make 6 (or 8 ) pills. Sign: One pill at 6 or 7
p. m. and one pill at 8 or 9 p. m.; a third pill may be taken at $10 \mathrm{p} . \mathrm{m}$. or later if needed.

15. Strong tincture of passiftora incarnata..f 3 ii 601

Dose: 10 to 30 drops in water every 10,20 or 30 minutes, as needed, to relieve distress in breathing. Four fluidrams must not be exceeded. Should drowsiness, vertigo or nausea occur, the medicine is to be discontinued.

It is only rarely that the last prescriptions will become necessary. Obviously such measures demand care in application and the indications for their use must be clear.

\section{GENERAL PRECAUTIONS.}

Those who are able to go to an immune region usually need no additional palliation. The less fortunate must, in addition to the use of one or more of the expedients detailed in the foregoing paragraphs, observe certain precautions. Exposure to dust, to smoke, to direct sunlight, to heat, must be avoided or minimized. Sometimes it is advisable to wear cotton filters in the nostril when exposed to the dust of city streets, when walking or driving on country roads, and especially during railway trips. Dark glasses will also be found extremely useful. Hurry and excitement are to be avoided, if possible. The bowels, the skin and the kidneys must be kept normally active or even a little stimulated.

Dict must be simple and on the whole sparing. Shellfish and elaborate mixtures are usually harmful. Meat is to be avoided or cut down to the lowest possible point. Pastries and sweets should be avoided. Concerning fruits, marked idiosyncrasies exist. Peaches, if pared, can usually be enjoyed, but the fuzzy skin distresses many persons. Strawberries eause aggravation of symptoms in a few hay-fever patients. Melons are not only palatable and refreshing, but have a slight diuretic action that is often helpful. A tepid bath, followed by a brisk cold rub, just before going to bed, and a hot sponge, followed by a brisk cold rub, in the morning, after the rest following pollantin or suprarenanal snuffing, will be found to conduce very much to comfort. Also advantage should be taken of every opportunity for rest and quiet.

By utilizing some or all of the palliative measures described, not in unintelligent routine, but varying the expedients with the individual and the occasion, the vast majority, probably at least nine-tenths of those hay-fever sufferers who are unable to take the best course-namely, flight to a region of peace-may be enabled to get through their season of tribulation with a minimum of discomfort, and even to enjoy, from time to time, hours and sometimes days of complete relief. In these intervals they have opportunity to realize the truth of the poet's utterance. "Tife's keenest pleasure is surcease of pain."

\section{THE, VALUE OF ROUTINE URINE EXAMINA- TION.}

A STATISTICAL INQUIRY.*

$$
\begin{aligned}
& \text { M. H. FUSSELL, M.D. } \\
& \text { PIILADELPHIA. }
\end{aligned}
$$

If an excuse for writing a paper of the above title is necessary, it may be found in an article published in The Journal of the American Medical Association, March 18-25, 1905, by Dr. Richard C. Cabot, Boston. The title of that paper is: "A Clinical Examination of the Urine. A Critical Study of the Commoner Methods."

The last two paragraphs of the paper are as follows: - The attempt to estimate the anatomic condition of the kidney by the measurement of albumin and the search for casts is fallacious in the extreme." "The most reliable data about the urine are those most simply and quickly obtained, the twenty-four hour quantity, the spe(ific gravity and the color."

To quote the title of the paper and these last para-

* Read by title before the Philadelphta County Medical Societr 
graphs alone is to make it appear that Dr. Cabot believes that clinical examination of the urine is "fallacious in the extreme."

As a matter of fact, Dr. Qabot attempts to prove by the investigation of a large number of urine examinations made at the Boston City Hospital, and a comparison of autopsy findings in the same cases, that albumin and casts in the urine do not always mean that a pathologic nephritis is present in the case, and, on the other hand, that a true pathologic nephritis may be present without the presence of albumin or casts in the urine. The paper is an extremely valuable one because it settles in a certain way that nephritis, in common with other diseases, may not always be surely diagnosed from one symptom alone, and because it calls renewed attention to the well-known fact that albumin and casts in the urine do not warrant the diagnosis of Bright's disease.

But I consider it an extremely dangerous paper to spread broad-cast among the medical profession. Many men will read the title and the conclusions alone and will jump to the opinion that if such an eminent authority as Dr. Cabot believes that Bright's disease can not be diagnosed by urine examination, then surely urine examinations are useless. It is true that a careful reading of the paper will not justify such an opinion, but I fear that will be the ultimate effect.

Everyone who has seen much of medical practice throughout this country, must realize with sincere regret that the more careful laboratory helps in diagnosis and treatment of disease, including urine examinations, are woefully neglected by the average practitioner of medicine. I fear that Dr. Cabot's paper will help to make the average man more careless, instead of helping him to make a more accurate diagnosis, as it surely was meant to do, and as it surely would do, if read carefully. The trouble is, I repeat, that it will be generally read by the title and the conclusions, and will thus give an erroneous idea of the author's meaning. Everyone knows that even though albumin and casts in the urine may not always mean nephritis, that their presence in the urine does mean that a vital organ is at fault, and this fault should be duly weighed in diagnosis, prognosis and treatment. Then, too, there are other conditions of the urine which are of the greatest importance, such as the presence or absence of glucose, of pus, of blood, persistently or intermittently large quantities of urine, etc., all of which should be made a note of in routine examinations.

During the past eighteen months I have kept notes of the color, specific gravity, albumin, sugar and microscopic findings of 1,666 urine examinations. Of these, 1,346 examinations showed entirely normal urine; 320 examinations showed something abnormal about the urine. The urine of 763 patients was examined; 669 patients had entirely normal urine; 94 had some abnormal sign in the urine.

It will be seen that approximately 20 per cent. of the urines examined were abnormal and about 12 per cent. of the patients whose urine was examined showed something abnormal in the urine.

If these abnormalities can be shown to have any effect of importance on either diagnosis, prognosis or treatment, surely then it is worth while to examine five urines to find one abnormal one. The work is of course, tedious, it takes time. but it assuredly can be done by the busiest practitioner and in my opinion it should be done and must be done if we are to advance in scientific methods. I have worked as a general practitioner long enough to test the value, and here make the appeal that every general practitioner provide himself with a private laboratory in which to work.

I have divided the cases which showed some abnormality into the following groups: First, 21 cases, showing either chemically or microseopically an abnormality which on further examination was found to be of no pathologic importance; second, 5 cases, showing albuminuria of pregnancy; third; 7 cases, showing acute nephritis (with apology to Dr. Cabot) ; fourth, 2 cases, showing urine of liysteria ; fifth, 4 cases, showing urine of diabetes meilitus; sixth, 2 cases, showing lactosuria during the beginning of lactation; seventh, 1 case, showing diabetes insipidus; eighth, 11 cases, in which there was severe arteriosclerosis with albumin and casts in the urine; ninth, 9 cases, showing true interstitial nephritis; tenth, 15 cases, acute conditions, developing during the course of the disease, severe albuminuria with casts and albumin; eleventh, 1 case, showing albumin caused by a beginning menstrual period; twelfth, 2 cases, in which there was cardiac disease with severe renal complications; thirteenth, 3 cases, in which there were chronic conditions, developing severe albuminuria and casts; fourteenth, 11 cases, showing a local condition of the genitourinary tract.

Now as to the 669 patients whose urine was found to be entircly normal, it was of the greatest value and comfort to know, for instance in a case of convulsions, that the urine was normal, and that the kidneys need not be taken into account in the treatment, or in a case of unconsciousness to know that I was not dealing with a uremic coma, indeed, it seems to me that of ten the negative finding is about as valuable as the positive, but it is the 94 abnormal cases that we want chiefly to consider.

I shall not cite all of these cases, of course, or I would be tiresome, but shall give only an abstract of a few of the imp.rtant ones. I desire here to state that I have in my possession the case histories of each of these 94 cases, so the pictures are not fanciful. Of the first group of 21 cases, thirteen patients were pregnant women showing a trace of albumin, but repeated examinations showed the albumin to be of no pathologic importance, and probably due to a leucorrhea. One case is worth quoting:

CASE 1.-A young single woman was rather suddenly seized with constant vomiting, severe headache, apparent delirium. The physician in attendance had discovered albumin in the urine. Careful examination showed the urine to be of small quantity-high specific gravity-with a trace of albumin. Mieroscopically there were no casts, but numerous leucocytes and epithelial cells. The urine examination did not justify the diagnosis of uremia, and a careful physical examination showed the patient to be pregnant.

This case proves Dr. Cabot's point, of the error of diagnosing Brights disease on insufficient grounds:

The tive cases of albuminuria of pregnancy showed the great importance of routine examinations, and $I$ fully believe that if no other cases needing treatment had been found save these five alone, that the whole work would have been justified. I believe the doctor is almost criminally neglectful who has a case of puerperal convulsions, in which he has had any opportunity of examining the urine and has not done so.

CAse 2.-Mrs. L., ii-para. With the first child there was considerable puerperal toxemia with albuminuria and casts in the urine. She became pregnant again in June, 1904, and immediately consulted me. Remembering her previous condition I urged a frequent urine examination. In July, 1904 the urine was normal. None was sent until November, 1904 , when $I$ calied at the home and urged her to send me a speci- 
men every week. The specimen sent at that time was mormal. She neglected to send another specimen. In the early part of December I was called and found her in convulsions with the urine full of albumin and casts. In spite of treatment she died in 48 hours.

T'his case not only proves that a urine examination is necessary, but that it should be made frequently in the later months of pregnancy.

The next group of seven cases of acute nephritis were all of the same character; none of them could, by any possibility, have been suspected as nephritic cases so early, had a urine examination not been made in a routine way. I believe all the patients were saved from a seriously prolonged illness, perhaps some from death.

Anyone can suspect nephritis when the individual has edema or a convulsion, but it is then frequently too late to lead the patient to health.

CAsE 3.-George H., married, a mill worker, called at my office complaining of malaise, aching limbs, headache, loss of appetite. The heart, lungs and abdomen were normal. The temperature was $101 \mathrm{~F}$. I supposed the case to be influenza and told the patient so, but ordered a specimen of urine.

Examination showed much albumin and many epithelial and blood casts. The case ran a typhoid course of acute nephritis.

Surely no diagnosis of nephritis could have been made at that time without a urine examination and surely it was only because the habit had been formed by me of making routine examinations that one was made. All the other cases were exactly of the same character.

The cases of diabetes mellitus and diabetes insipidus and of lactosuria were of the greatest importance. None of them would certainly have been diagnosed had I not made a habit of examining the urine regularly.

CASE 4.-Diabetes mellitus. Otto D. was seized suddenly with pneumonia. Examination of the urine showed 5.5 per cent of grape sugar. Questioning revealed the fact that for some months previous to his attack of pneumonia there har been the classical symptoms of diabetes mellitus. These symptoms had been disregarded and the routine examinations were the first to reveal the presence of sugar. 'A particularly unfavorable prognosis was made, which was upheld by the unfortunate termination of the case.

CASE 5.-Diabetes insipidus. Charles J. came to my office complaining of thirst, pain in the back and polyuria. Examination showed a large quantity of urine-eleven quarts in 24 hours, having a specific gravity of 1,001 and free from albumin, casts or sugar.

CASE 6.-Lactosuria. Mrs. L., iv-para. On the third day of the puerperium the urine, which was being examined for albumin and casts which had been long present, showed the presence of sugar by Fehling's test. Careful subsequent examinations showed this reducing agent to be some other body than grape sugar, and in all probability lactose. It disappeared from the urine in a few days.

No amount of speculation could have made a differential diagnosis in those cases without a urine examination.

The group of arteriosclerosis cases shows, perhaps, the value of Dr. Cabot's paper equally as well as the next group of true cases of nephritis; and at the same time it shows, I think, the great value of examining the urine of every patient coming under our care. In order to draw the line between the cases of arteriosclerosis with albumin and casts in the urine, and the true cases of nephritis with arteriosclerosis, the following points should be kept in mind:

First, almost all cases of arteriosclerosis have albumin and casts in the urine. but the urine is usually of normal quaritity and specific gravity. The heart shows signs of myocarditis, while the symptoms are those referable to general arteriosclerosis, rather than to a uremic condition.
On the other hand, the true cases of interstitial nephritis usually have large quantities of urine of low specific gravity with considerable hypertrophy of the heart and marked accentuation of the second aortic sound with eye symptoms, or attacks which are truly uremic.

CASE 7.-Mrs. R., aged 64, had extensive arteriosclerosi: much dyspnea, and irregular heart action. On many occasion. the urine of this patient showed a trace of albumin and a few casts, but always the symptoms were dyspnea and cardiac palpitation. At no time were there uremic symptoms.

CASE 8.-Mrs. S. had marked arteriosclerosis, retinitis and hypertrophy of the left heart. The urine was always of low specific gravity with constant albumin and pale, granular casts Uremic symptoms developed and she died in convulsions.

The group of acute cases developing albumin and casts shows the absurdity of treating acute conditions: without repeated urine examinations. The same mar be more forcibly said of chronic cases.

Every careful practitioner has seen serious complications arise in acute and chronic conditions. the cause of which was entirely unsuspected until the urine was examined. If the practitioner had been in the habit of examining the urine at regular intervals, the cause of the untoward symptoms would have been found before these symptoms became a serious complication.

As an instance of the first of these:

CASE 9.-A case of pneumonia. The patient, D. F., had a very severe attack of pneumonia of the right lung, with in. creasing dullness of mind. Examination of the urine showed a gradually increasing amount of albumin and numerous casts showing the severity of the toxemia. The prognosis was guarded and the termination showed its propriety.

CASE 10.-Mrs. H., in course of an ordinary case of tuberculosis of the lungs, became suddenly unconscious. Examination showed the urine to contain albumin enough to cause it to become solid on boiling. She died of uremia. The presence of nephritis was not suspected because the urine had not been regularly examined.

The last group of local genitourinary cases show equally as well as the others the great importance, indeed the absolute necessity of a urine examination before a diagnosis even can be made, not to speak of treatment. This group contains two cases of pyuria due to stone. four cases of acute renal colic with passage of a small calculus, one case of cystitis and four cases of hematuria.

How futile even a guess in these cases would have been and how easily the diagnosis was cleared up by the microscope is shown by Case 11 .

CASE 11.-A young man was suddenly seized with pain and tenderness in the region of the appendix. Tenderness and mus. cular resistence were extreme. Very frequent urination attracted attention to the urine. There was free blood in the urine. In a few hours a small renal calculus was passed This patient might easily have been operated on for appen. dicitis had a urine examination not been made.

I therefore claim, as proven by these cases, which, of course, are not unique, that a urine examination is of the utmost value indeed, and that a tentative diagnosis only can be made without it in as many as 12 per cent. of all cases treated.

I further claim that the examination should be routine. To examine the urine only when symptoms point to the genitourinary tract is to miss the diagnosis in many important cases, and often if the examination is made only after such symptoms appear it is too late to intervene for the patient's recovery.

I again call attention, therefore, to the absolute necessity of a clinical laboratory at each practitioner's commano, if he desires to gire full ralue of his service to his patients. 